\title{
H-infinity PID controller for a Ball and Beam System
}

\author{
Akhil Jose ${ }^{1}$, Avinashe K K ${ }^{2}$, Dhanoj $M^{3}$, E.S. Yadav ${ }^{4}$ \\ Assistant Professor, E\&I Department, VJEC, Chemperi Kerala, India ${ }^{1}$ \\ Assistant Professor, E\&I Department, VJEC, Chemperi Kerala, India ${ }^{2}$ \\ Assistant Professor, E\&I Department, VJEC, Chemperi Kerala, India ${ }^{3}$ \\ M.Tech., Control Systems, MIT,Manipal, Karnataka ${ }^{4}$
}

\begin{abstract}
Ball and beam system is a benchmark system to study various control algorithms. It consists of a beam that can be tilted by a DC servo motor and a ball rolling back and forth on the top of the beam. The main reason for its acceptance in labs across the globe is its property that it is open loop unstable. It can serve as a conventional tool for implementation of many classical and modern control system design method.

In the paper, the theory of H-infinity control is used to design a control system for the ball and beam system. The theory of Robust control is extended to design a robust PID controller. Controller parameter values for PID controller i.e. gains are found from the robust controller designed.
\end{abstract}

Keywords: Robust controller, Robust PID, H-infinity, Ball and Beam.

\section{INTRODUCTION}

This In this paper, the problem of controlling the ball and beam system is considered. The significance of the ball and beam system is that it is a simple system which is open-loop unstable. This research is devoted to the problem of synthesizing proportional-integral-derivative (PID) controllers for robust stability and performance of the ball and beam system.

Robust control considers the design of decision or control rules that fare well across a range of alternative models. Thus robust control is inherently about model uncertainty, particularly focusing on the implications of model uncertainty for decisions [1]. Robust control allows policymakers to formulate policies that guard against model misspecification. The precision with which economic models can be expressed mathematically belies the fact that they cannot claim to be anything more than approximations to an unknown, and possiblyunknowable, data-generating process. This unfortunate reality means that economic decisions are inevitably made in situations where important aspects of the environment are cloaked, hidden behind a cloud of uncertainty. While such uncertainty is hardly welcome, it need not render decision makers powerless, as its effects can in principle be mitigated through the application of robust control methods. Robust control provides a set of tools to assist decision makers confronting uncertainty who are either unable or unwilling to specify a probability distribution over possible specification errors [2].

A basic desideratum for robust control in practice is that the system remains stable in the face of perturbations, and since instability may be equated with infinite loss, minimizing the worst case outcomes will insure stability. In robust PID controller design, the result from the robust control is used to find the set of all parameters KI, KP, KD that simultaneously places all the roots into specific region in the complex plane [3]. 


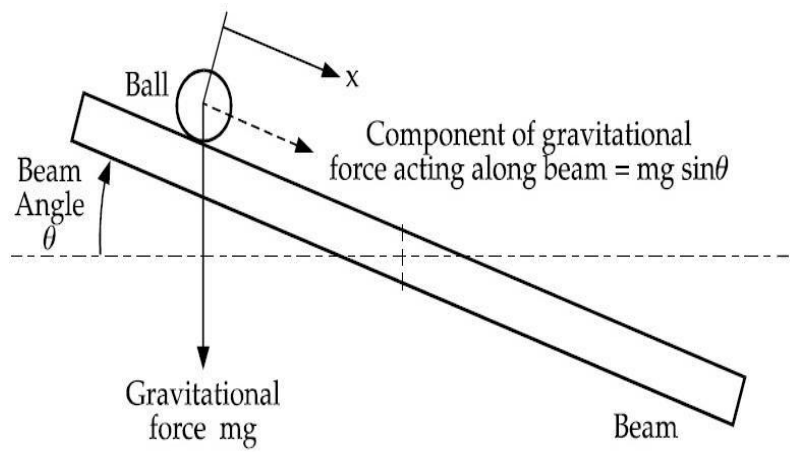

Fig. 2 Ball dynamics

The figure 2 shows the ball dynamics of ball and beam system. Various parameters that are taken into consideration for the modelling of system are:

Mass of the ball $(\mathrm{m})=0.011 \mathrm{~kg}$

Radius of the ball $(\mathrm{R})=.015 \mathrm{~m}$

Gravitational constant $(\mathrm{g})=-$

$9.8 \mathrm{~ms}-2$ Length of the beam $=$

$0.4 \mathrm{~m}$

Radius of the gear $(\mathrm{d})=$

$0.04 \mathrm{~m}$ Ball position co-

ordinate $=\mathrm{r}$ Beam angle $=$

$\alpha$

Servo gear angle $=\theta$

Also the moment of inertia of the ball is given by:

$$
I=\frac{2 m r^{2}}{5}
$$

Now Lagrange's equation for motion of the ball is:

$$
\left(\frac{J}{R^{2}}+m\right) r^{\alpha}+m g \sin \propto-m \dot{r}(\dot{\alpha})^{2}=0
$$

Then linearize the equation about the beam angle ' $\alpha$ ' equal to zero. Again ' $\alpha$ ' being very small, the equation which relates beam angle to the gear can be approximated by

$$
\alpha=\frac{d}{L} \theta
$$

From this we get the following equation:

$$
\left(\frac{1}{R^{2}}+m\right) \ddot{r}=-m g \frac{d}{l} \theta
$$

Taking Laplace transform of the above equation and rearranging we find the transfer function for the gear angle $\theta$ (s) to ball position $R(s)$.

$$
\frac{R(s)}{\theta(s)}=\frac{-m g d}{L s^{2}\left(\frac{J}{R^{2}}+m\right)}
$$

Substituting the values in 3 , we get

$$
P(s)=\frac{R(s)}{\theta(s)}=\frac{0.7}{s^{2}}
$$

This is the final function for the model

\section{III.DESIGN OF ROBUST RID CONTROLLER}

H-infinity control criterion is applied to design the PID controller for the ball and beam system. The robust stability and performance of the general system is analysed. Based on that the internal stability of the system is checked and controller is designed. The design method proposes a method based on for $\mathrm{H}$-infinity design of PID controller for ball and beam system.

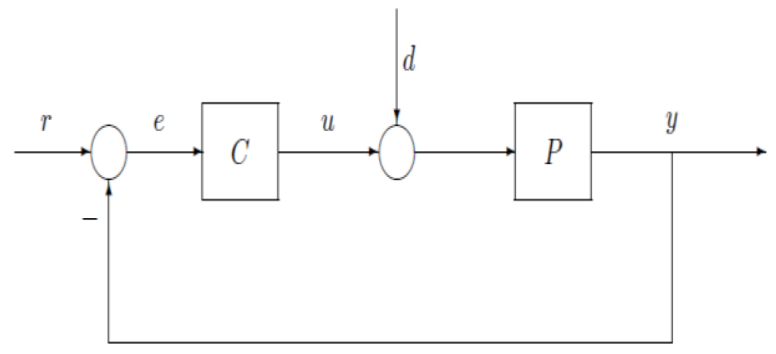

Fig. 3 Unity feedback control loop with disturbance.

The figure 3 shows a unity feedback control loop where $C$ is the controller and $\mathrm{P}$ is the plant. Let $Q$ represent the controller of internal model control structure. The unity feedback loop can be equivalent to an internal model control structure through

$$
C=\frac{Q}{1-P Q}
$$

So far, most of design methods are developed aiming at integrator/dead time processes for PID controllers, as well as Smith predictors. This limits the use of controllers.

As considered earlier the ball and beam plant transfer function of the plant $\mathrm{P}(\mathrm{s})$ without considering the uncertainty,

$$
P(s)=\frac{0.7}{s^{2}}
$$

The actual plant transfer function of the plant is With an uncertain time delay

$$
\widetilde{P}(s)=e^{-\tau s} \frac{0.7}{s^{2}}
$$

Using first order Taylor formula we approximate time delay as $(1-\tau \mathrm{s})$, we obtain the new plant transfer function as: 


$$
P=\frac{0.7(1-\tau s)}{s^{2}}
$$

Under the nominal condition, the sensitivity of closed loop system can be expressed as

$$
S=\frac{1}{1+P C}=1-P Q
$$

Where $\mathrm{Q}(\mathrm{s})$ is defined as:

$$
Q(s)=\frac{C(s)}{1+C(s) P(s)}
$$

Now we regard it as the nominal process model for developing the analytical design formula of the controller $\mathrm{C}(\mathrm{s})$. In fact, the introduced model error can be regarded as a part of the process uncertainty. According to the robust control theory [8], the controller $\mathrm{C}(\mathrm{s})$ can ensure the closed-loop system if and only if

$$
\left\|\Delta_{m}(s) T(s)\right\|_{\infty}=\left\|\Delta_{m}(s) \frac{C(s) P(s)}{1+C(s) G(s)}\right\|_{\infty}<1
$$

$\left|\Delta_{m}(s)\right|$ defines the multiplicative uncertainty of the process.

The controller structure and parameters are derived from an optimal performance criterion simultaneously. We are designing the controller in terms of $\mathrm{H}$-infinity optimal criterion $\min \|W(s) S(s)\|_{\infty}$, where $\mathrm{W}(\mathrm{s})$ is a weight function that is used for normalizing the system input $\mathrm{r}(\mathrm{s})$ as a unit impulse signal. $H(s)$ denotes the transfer function matrix from $r$ and $d$ to $y$ and $u$. i.e.

$$
H=\left[\begin{array}{cc}
P Q & P(1-P Q) \\
Q & -P Q
\end{array}\right]
$$

The closed-loop system is said to be internal stable if all the transfer functions in $H(s)$ are stable.

Actually the system can be said internally stable if and only if $\mathrm{Q}(\mathrm{s}), \mathrm{Q}(\mathrm{s}) * \mathrm{P}(\mathrm{s})$ and $\mathrm{P}(\mathrm{s}) *(1-\mathrm{Q}(\mathrm{s}) * \mathrm{P}(\mathrm{s}))$ (i.e. $\mathrm{P}(\mathrm{s}) * \mathrm{~S}(\mathrm{~s}))$ are stable and proper transfer functions. In view of that $\mathrm{P}(\mathrm{s})$ is unstable here, construct $\mathrm{Q} 1(\mathrm{~s})$ to let $\mathrm{Q}(\mathrm{s})=$ sQ1(s) so that Q1(s) stability can ensure the stability of $\mathrm{Q}(\mathrm{s})$ and $\mathrm{Q}(\mathrm{s})^{*} \mathrm{P}(\mathrm{s})$. At the same time, if $\mathrm{S}(\mathrm{s})$ (i.e. 1$\left.\mathrm{Q}(\mathrm{s})^{*} \mathrm{P}(\mathrm{s})\right)$ is made stable and possesses at least one zero at $\mathrm{s}=0$, the closed-loop system is surely to be internally stable.

In process control $\mathrm{W}(s)$ can be selected as $1 / s$, which implies the system input is a unit step signal. Note that $P(s)$ has a zero $s=1 / \tau$ in the right half plane. In terms of the well-known maximum modulus theorem [6], there is

$\|W(s) S(s)\|_{\infty}=\| W(s)\left(1-P(s) Q(s) \|_{\infty} \geq|W(1 / \tau)|\right.$ Minimizing the left-hand side the inequality yields

$$
\min \|W(s)(1-P(s) Q(s))\|_{\infty}=\left|W\left(\frac{1}{\tau}\right)\right|
$$

By the analysis of the above equation we obtain the optimal Qim(s) i.e.

$$
Q_{i m}(s)=\frac{W(s)-\tau}{W(s) \cdot s P(s)}
$$

Therefore optimal $\mathrm{Q}(\mathrm{s})$ can be obtained as follows:

$$
Q_{i m}(s)=\frac{s^{2}}{k}
$$

Obviously $\mathrm{Q}_{\mathrm{im}}(\mathrm{s})$ is improper and cannot be realized in practice. By employing a low-pass filter $\mathrm{J}(\mathrm{s})$, the suboptimal $Q(s)$ can be obtained as $Q(s)=Q_{i m}(s) J(s)$.

In fact, most control systems in chemical industry aim at the process constant value output, which requires that the closed-loop system output gradually reach the set point value

$$
\lim _{s \rightarrow 0} S(s)=\lim _{s \rightarrow 0} 1-P(s) Q(s)=0
$$

' $s$ ' tends to zero means $t$ tends to infinity (final value theorem), so that the process results in constant output[12] The sensitivity function $\mathrm{S}(\mathrm{s})=1-\mathrm{Q}(\mathrm{s}) \cdot \mathrm{P}(\mathrm{s})$ should possess at least 3 zeros at $\mathrm{s}=0$ to reject the load disturbance injected at the process input side. Thus we select $\mathrm{J}_{\mathrm{c}}(\mathrm{s})$ in form of

$$
J_{c}(s)=\frac{a s^{2}+b s+1}{\left(\lambda_{c} s+1\right)^{4}}
$$

Where $\lambda_{c}$ is the tuning parameter.

Considering that $S(s)$ should be composed of those terms that $s^{3}$ or $s$ with index over 3 , we can figure out $a=6 \lambda_{c}^{2}+4 \lambda_{c} \tau+\tau^{2}, b=4 \lambda_{c}+\tau$.

Therefore $\mathrm{Q}(\mathrm{s})$ is obtained as

$$
Q(s)=Q_{i m}(s) J_{c}(s)
$$

$$
Q(s)=\frac{s^{2}\left[\left(6 \lambda_{c}^{2}+4 \lambda_{c} \tau+\tau^{2}\right) s^{2}+\left(4 \lambda_{c}+\tau\right) s+1\right]}{k\left(\lambda_{c} s+1\right)^{4}}
$$

And $\mathrm{C}(\mathrm{s})$ is in form of

$$
\begin{aligned}
& C(s)=\frac{Q(s)}{1-Q(s) P(s)} \\
& =\frac{1}{k} \cdot \frac{\left(6 \lambda_{c}^{2}+4 \lambda_{c} \tau+\tau^{2}\right) s^{2}+\left(4 \lambda_{c}+\tau\right) s+1}{\lambda_{c}^{4} s^{2}+\left(4 \lambda_{c}^{3}+6 \lambda_{c}^{2} \tau+4 \lambda_{c} \tau^{2}+\tau^{3}\right) s}
\end{aligned}
$$

This is PID controller. Suppose that the PID controller structure is given by

$$
C(s)=k_{c}\left(1+\frac{1}{T_{i} s}+\frac{T_{d} s}{T_{f} s+1}\right)
$$

The parameters can be written as

$$
\begin{gathered}
k_{c}=\frac{T_{i}}{k\left(4 \lambda_{c}^{3}+6 \lambda_{c}^{2} \tau+4 \lambda_{c} \tau^{2}+\tau^{3}\right)} \\
T_{f}=\frac{\lambda_{c}^{4}}{4 \lambda_{c}^{3}+6 \lambda_{c}^{2}+4 \lambda_{c} \tau^{2}+\tau^{3}} \\
T_{d}=\frac{6 \lambda_{c}^{2}+4 \lambda_{c} \tau+\tau^{2}}{T_{i}}-T_{f}
\end{gathered}
$$




$$
T_{i}=4 \lambda_{c}+\tau-T_{f}
$$

In ideal situation, the set point filter $\mathrm{F}(\mathrm{s})$ is employed for the ideal optimal closed-loop transfer function $T_{i n}(s)=$ $e^{-\tau s}$, which requires the filter form of

$$
F_{\text {in }}(s)=\frac{\left(\lambda_{c} s+1\right)^{4}}{\left(6 \lambda_{c}^{2}+4 \lambda_{c} \tau+\tau^{2}\right) s^{2}+\left(4 \lambda_{c}+\tau\right) s+1}
$$

So that $T_{\text {in }}(s)=F_{\text {in }}(s) Q(s) P(s)=e^{-\tau s}$. It is the ideal optimal closed-loop transfer function since the closed-loop system output can accurately track the set point value just after the process time delay $\tau$. However $\mathrm{F}(\mathrm{s})$ is not a proper function that can be physically realized. The lowpass filter $\mathrm{J}(\mathrm{s})$ added will roll off it at high frequency. For simplicity we select $J_{f}(s)$ in form of

$$
J_{f}(s)=\frac{1}{\left(\lambda_{f} s+1\right)^{2}}
$$

Where, $\lambda_{f}$ is the tuning parameter. Hence $\mathrm{f}(\mathrm{s})$ is obtained as

$$
\begin{aligned}
& F(s) \\
& =\frac{\left(\lambda_{c} s+1\right)^{4}}{\left[\left(6 \lambda_{c}^{2}+4 \lambda_{c} \tau+\tau^{2}\right) s^{2}+\left(4 \lambda_{c}+\tau\right) s+1\right]\left(\lambda_{f} s+1\right)^{2}}
\end{aligned}
$$

Generally we prefer to let $\lambda_{f}=\lambda_{c}$ so that $\mathrm{F}(\mathrm{s})$ can be simplified as

$$
F(s)=\frac{\left(\lambda_{c} s+1\right)^{2}}{\left[\left(6 \lambda_{c}^{2}+4 \lambda_{c} \tau+\tau^{2}\right) s^{2}+\left(4 \lambda_{c}+\tau\right) s+1\right]}
$$

Now we have the controller and the PID values. Consider the same time delay value we used in the previous control problem. i.e. $\tau=0.1$.

In the proposed method [12] take $\lambda_{f}=\lambda_{c}=2.5 \tau$. Hence we get $\lambda_{c}=0.25$. By substituting these values in above equations we obtain the following results:

$T_{f}=0.036, T_{i}=1.064, T_{d}=0.456, K_{c}=13.694 \mathrm{and}$

$$
F(s)=\frac{(0.25 s+1)^{2}}{0.485 s^{2}+1.1 s+1}
$$

Further studies on the plant response to the controllers found above were done successfully and the results are discussed in the following chapter. Step response was given as input and response of the system with both controllers with and without filter has been studied.

\section{IV.RESULTS AND CONCLUSION}

From the H-infinity synthesis using maximum modulus theorem a robust controller was designed then PID controller parameters are obtained from the controller and their values also are found. Furthermore $\mathrm{H}$-infinity PID controller response is obtained with the help of MATLAB commands. The output response obtained is given in following figures

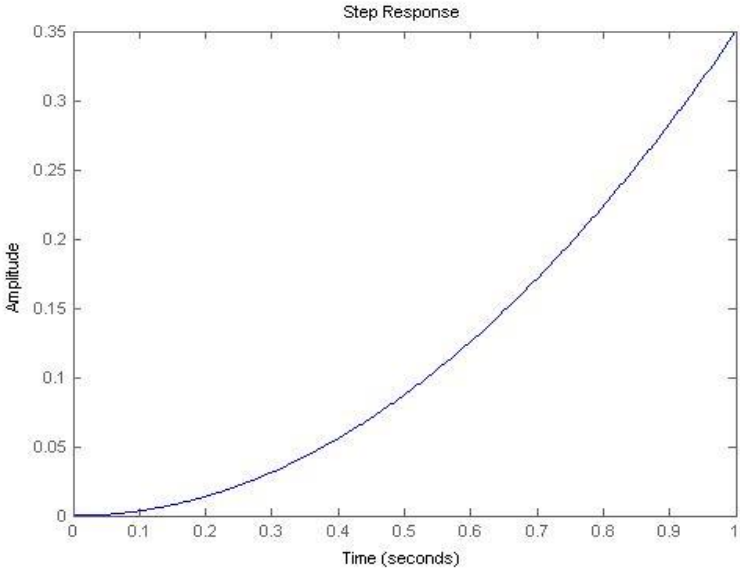

Fig 4 System response for a unit step input

From figure 4 we can see the system instability clearly. Figure 5 and figure 6 shows the responses of the systems with robust controller and robust PID controller

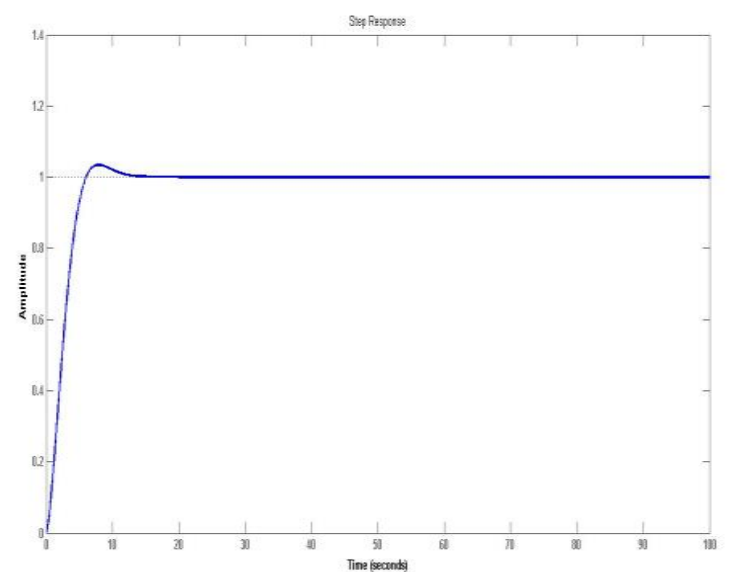

Fig. 6 Step response of plant using robust controller with filter.

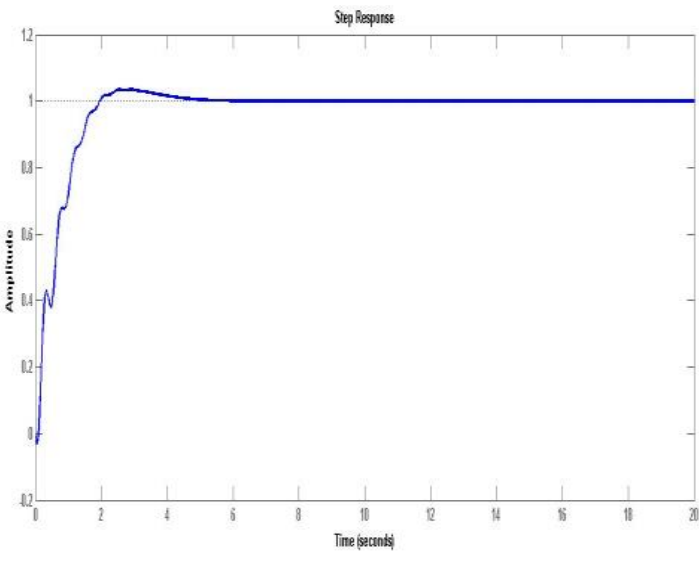


Fig. 6 Step response of plant using robust PID controller with filter.

Comparisons of the Results from Various Controllers

We have discussed about the results obtained by using different controllers. It's clearly observable from the step responses that controllers behaved differently with the plant. Thus a comparison of the results we obtained from all the controllers must be proper to complete our study about the controllers and results.

\begin{tabular}{|l|c|c|c|c|}
\hline $\begin{array}{l}\text { Controller } \\
\text { Type }\end{array}$ & $\begin{array}{l}\text { Rise } \\
\text { time(sec }\end{array}$ & $\begin{array}{l}\text { Settling } \\
\text { time }\end{array}$ & $\begin{array}{l}\text { Overshoo } \\
\text { t }(\%)\end{array}$ & $\begin{array}{l}\text { Peak } \\
\text { amplitud }\end{array}$ \\
\hline $\begin{array}{l}\text { Robust } \\
\text { Controller }\end{array}$ & 5.922 & 10 & 3.43 & 1.03 \\
\hline $\begin{array}{l}\text { Robust PID } \\
\text { controller }\end{array}$ & 1.32 & 3.71 & 3.45 & 1.03 \\
\hline
\end{tabular}

Comparison of the response parameters for both robust and robust PID controllers is given in the table 1. From the table and the graph it's evident that both the controllers showed very good control performance.

\section{REFERENCES}

[1] Noah Williams, "Robust control: An Entry for the New Palgrave", Department of Economics, Princeton University, 2nd Edition.

[2] Richard Dennis, Kai Leitemo and Ulf Soderstrom, "Methods for Robust Control", Federal reserve of San Francisco working paper series 2006-10"'.

[3] Ming-Tzu Ho and Sheng-Tsai Huang, "Robust PID Controller Design for Plants with Structured and Unstructured Uncertainty", Proceedings of the 42nd IEEE Conference on Decision and Control, Maui, Hawaii USA,.

[4] Peter E Wellstead, "Ball and Beam1: basics", control system principles.co.uk

[5] Morari, M., \& Zafiriou, E. (1989). "Robust process control". Englewood Cliffs, NJ: Prentice Hall.

[6] Doyle, J.C. and Francis, B.A. et al. "Feedback Control Theory". Macmillan Publishing Company, 1992

[7] Tao Liu, Danying Gu, Weidong Zhang "A H infinity design method of PID controller for second-order processes with integrator and time delay", Proceedings of the 42nd IEEEConference an Decision and Control Mad, Haraii USA, December 2003. 